\title{
Gerontology
}

Gerontology 2015;61:268-280

DOI: $10.1159 / 000363698$
Received: November 4, 2013

Accepted: May 19, 2014

Published online: October 22, 2014

\section{Towards a Future Robotic Home Environment: A Survey}

\author{
Jörg Güttler Christos Georgoulas Thomas Linner Thomas Bock \\ Building Realization and Robotics Lab, Department of Architecture, Technical University Munich, Munich, Germany
}

\section{Key Words \\ Ambient integrated robotics $\cdot$ Architecture $\cdot$ Ergonomic furniture · Kitchen design · Assistive mobility demographic change $\cdot$ Vision system $\cdot$ Activities of daily living}

\begin{abstract}
Purpose: Demographic change has resulted in an increase of elderly people, while at the same time the number of active working people is falling. In the future, there will be less caretaking, which is necessary to support the aging population. In order to enable the aged population to live in dignity, they should be able to perform activities of daily living (ADLs) as independently as possible. The aim of this paper is to describe several solutions and concepts that can support elderly people in their ADLs in a way that allows them to stay self-sufficient for as long as possible. Method: To reach this goal, the Building Realization and Robotics Lab is researching in the field of ambient assisted living. The idea is to implement robots and sensors in the home environment so as to efficiently support the inhabitants in their ADLs and eventually increase their independence. Through embedding vital sensors into furniture and using ICT technologies, the health status of elderly people can be remotely evaluated by a physician or family members. By investigating ergonomic aspects specific to elderly people (e.g. via an age-simulation suit), it is possible to develop and test new concepts and novel applications, which will offer innovative solutions. Via the
\end{abstract}

introduction of mechatronics and robotics, the home environment can be made able to seamlessly interact with the inhabitant through gestures, vocal commands, and visual recognition algorithms. Meanwhile, several solutions have been developed that address how to build a smart home environment in order to create an ambient assisted environment. This article describes how these concepts were developed. The approach for each concept, proposed in this article, was performed as follows: (1) research of needs, (2) creating definitions of requirements, (3) identification of necessary technology and processes, (4) building initial concepts, (5) experiments in a real environment, and (6) development of the final concepts. To keep these concepts costeffective, the suggested solutions are modular. Therefore, it will be possible to straightforwardly install the proposed devices in an existing home environment in a 'plug and play' manner once the terminals can be prefabricated off-site. $\boldsymbol{R e}$ sults and Discussion: This article shows a variety of concepts that have been developed to support elderly people in their ADLs. The prototypes of the proposed concepts in this paper have been tested with elderly people. The results of the tests show that robots embedded in furniture, walls, ceiling, etc. offer enhanced support, properly addressing elderly as well as disabled people to individually and independently manage their ADLs. In order to make the concepts realizable in terms of cost, it will be necessary to standardize and modularize these concepts for industrial fabrication.

(c) 2014 S. Karger AG, Basel

Thomas Bock

Building Realization and Robotics Lab,

Department of Architecture, Technical University Munich

DE-80333 Munich (Germany)

E-Mail thomas.bock@br2.ar.tum.de 


\section{Introduction}

In the past, the population pyramid between young and elderly people was totally different than nowadays. Industrialization has greatly affected birthrates, causing the 'demographic change', in which the elderly population has grown rapidly. It is expected that the elderly population will increase from $7 \%$ (in 2000) to $16 \%$ within the next 50 years [1].

Elderly people face a lot of challenges in performing their activities of daily living (ADLs) [2]. ADLs are tasks which usually need to be performed independently. Considering the demographic change, ADL problems will also increase in the coming years. Therefore, the Building Realization and Robotics Lab is focusing on the development of ambient assisted living (AAL) environments.

In this paper, concepts which will effectively assist elderly people in accomplishing their ADLs independently are summarized. As several ceiling suspended lift systems are already on the market for this kind of use [3], the first concept will describe a multirobotic assistance system (MRAS) [4]. Then the development of a robotic furniture system will be described [5]. Additionally, novel furniture concepts such as a robotic kitchen [6], an optimized wheelchair design [7], and a robotic bathroom [8] will be described. At the end, an overview based on advanced building engineering [9] is provided to show further research goals of the Lab.

\section{Multirobotic Assistant System}

The MRAS [4] (fig. 1,2) concept presents a multirobotic system that is mounted on the ceiling and thus can meet ergonomics aspects. Through an air purifier and automatic ambient light control, the proposed system is able to provide an adequate environment for sleeping. An automated pill dispenser module ensures that the user will get medication at the proper dose and time. With flexible wires, which can be used through grabbing or through being attached to the individual with a harness, this application can assist elderly people in their functional transfers by supporting them as they go from a sitting to a standing position and vice versa. For the realization of this application, four concepts have been defined: technology, modularity, exchangeability, and low maintenance.

In the future, the independence of elderly people can be improved and thereby the need for caretakers reduced through such technologies and several further future ad-

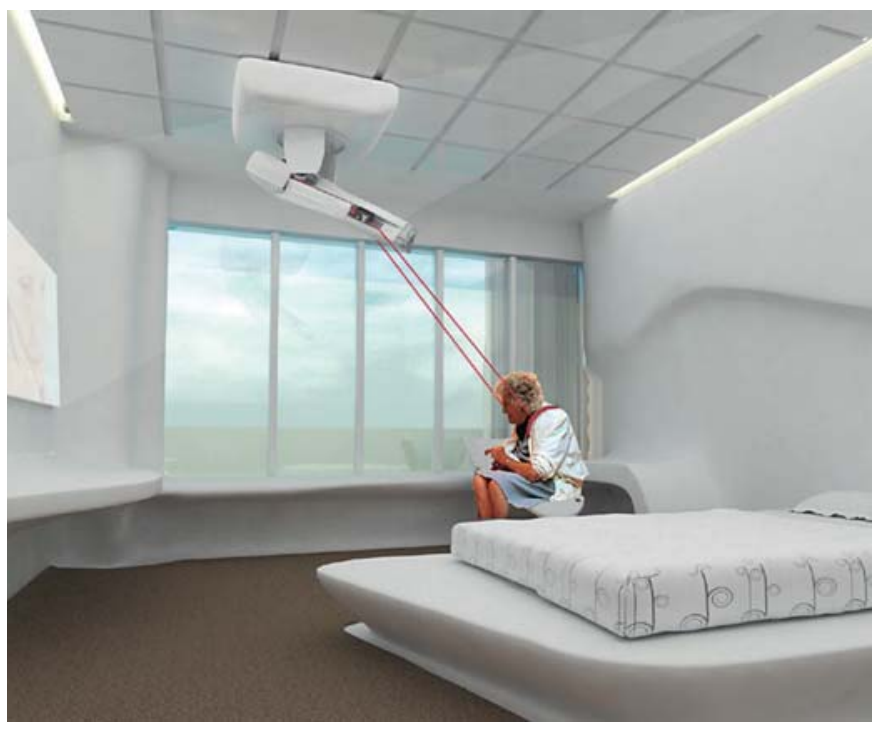

Fig. 1. Functional transfer assistant [4]. With permission, Christian Thuesen.

ditional features. The device will be modular so that delivery and, depending on user need, updating and exchange will be more cost-efficient. The exchangeability will be realized by a 'plug and play' system, which will also be beneficial for installation. To avoid hassle between customers and manufactures, the maintenance will be as low as possible, so that the application is efficient.

To comply with these requests, this application is divided into three main components: the main grid, the main actuator, and the main body. To allow the device mobility, steel rails arranged in a rectangular mesh will allow the main actuator to move to all possible locations. The main actuator is the heart of the application and is responsible for the mobility, information exchange with other all subsystems, and charging at a predefined location in the main grid. A ball-bearing arrangement at all intersection points was designed to allow the main actuator to progress on the main grid.

The main body will be attached to the main actuator, and thus can offer all the functionalities and service deliveries. As it is exchangeable, the request of modularity and easy installation is fully addressed. The aim of this application is to increase the independence of elderly people so they can spend more time in their own home environment.

A lighting system will be integrated, which will be controlled by the main control module. Thereby, the light will be offered in areas where low lighting conditions are detected. Depending on the time of day, the light color will also be regulated. 


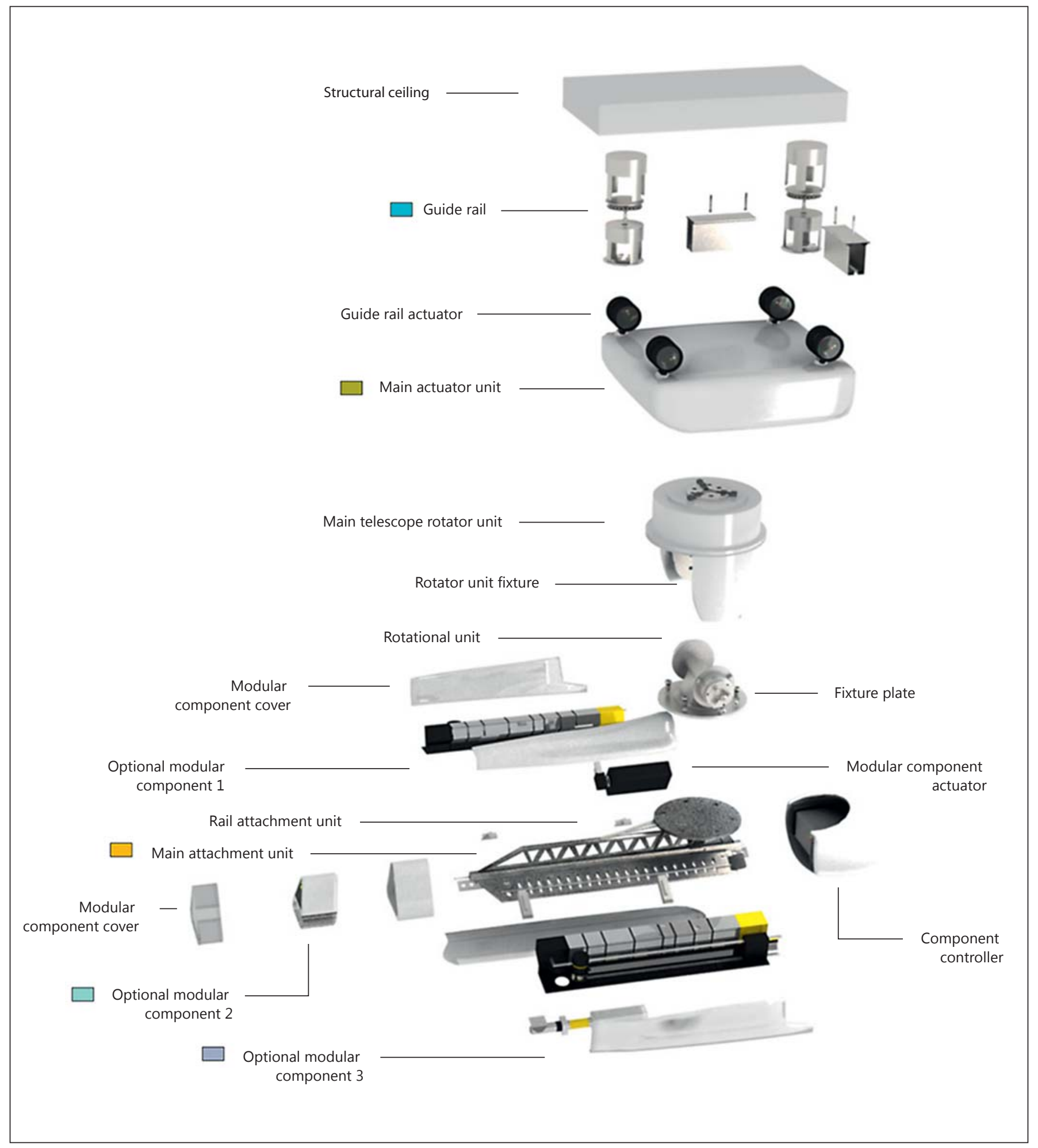

Fig. 2. Proposed system overview [4]. With permission, Christian Thuesen. 


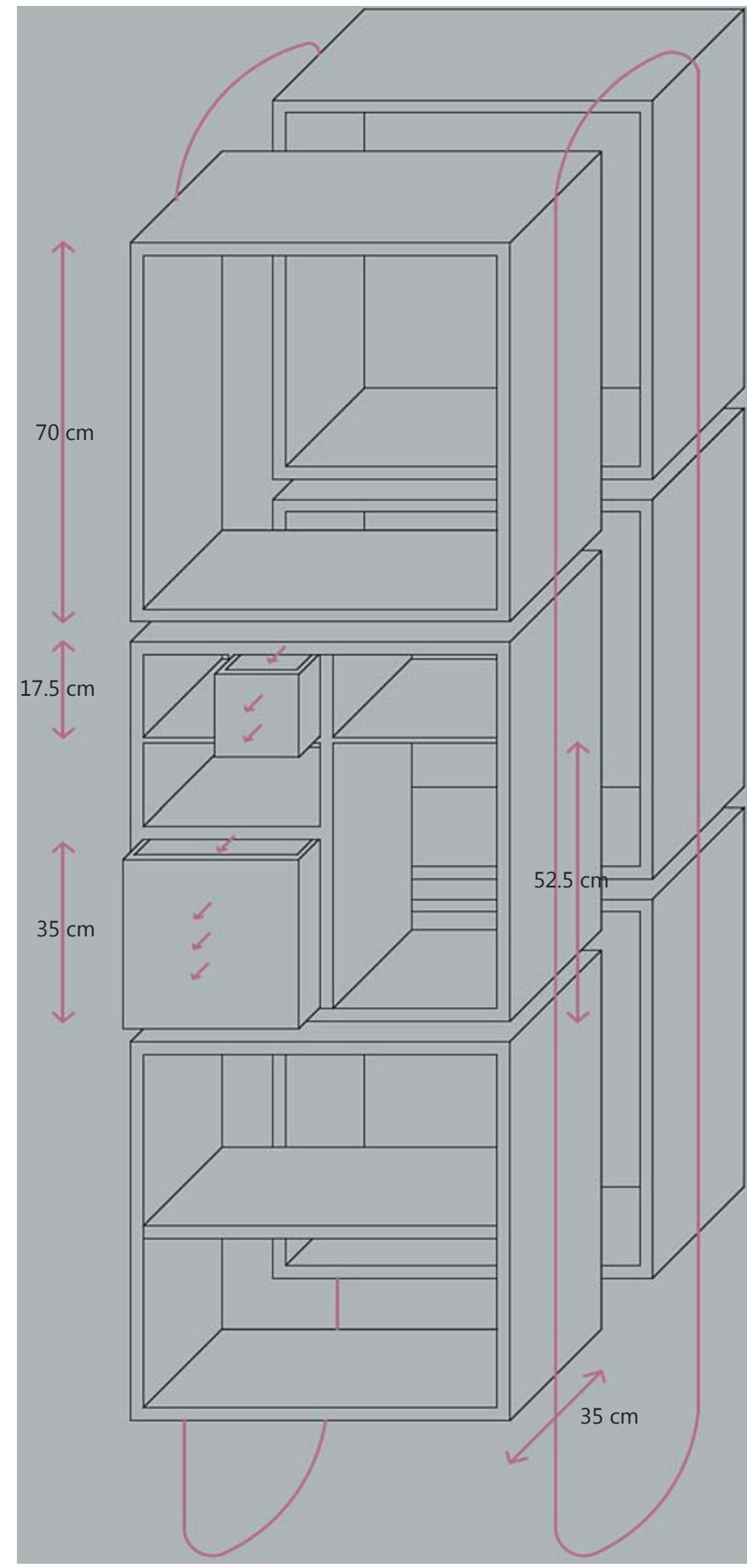

Fig. 3. Rotary motion path [5]. With permission, Susanne Meyer.
Through the use of active noise control and antiphasing technology, repetitive noise patterns can be reduced, leading to a quieter environment.

A DIY (do-it-yourself) healthcare system will be established - one that will be able to measure passive blood pressure, offer telecare and teleconsulting by sending the data via a communication network to a physician, and inject medicine by a needleless injection feature through a solid microneedle system attached to the skin. Also a medication dispenser, which will collect the necessary medicine automatically and dispense these properly to the user, has been proposed.

To support the user with mobility, a harness can be used. Once the user mounts the harness on his body, functional transfer assistance can be realized by lifting the user from a sitting position to a standing position, or to transfer the user in a convenient way.

A vision system can detect the presence of the user and autonomously navigate the main body on the grid by creating stereo vision of the acquired optical scene [10]. The rapid improvement of computer technology has made many real-time applications possible. Combined with a voice recognition module, the response performance of the MRAS device can be greatly increased in order that also elderly people with movement disabilities are able to use the device [11].

\section{Vision-Controlled Robotic Furniture System}

There are already proposed solutions for integrating sensors actuators and robots into the home environment (e.g. Robotic Rooms [12], Wabot House [13], and Robot Town [14]) to support elderly people in their ADLs. However, these solutions have only been tested in a laboratory environment and have not been put to use in a mediumsized apartment. The Vision Controlled Robotic Furniture System [5] focuses on offering a compact ergonomic system that can be easily installed without major modifications - also in smaller apartments. As the necessary apartment size for a single person is expected to increase to $55 \mathrm{~m}^{2}$ by 2030 , the proposed approach aims to simplify ADLs while at the same time reducing the space utilization requirement.

Ergonomy is thus a very important requirement for this application. Ergonomy is the science of getting the best of something with the smallest effort. In addition, aesthetics is given the same importance as functionality. In order to be ergonomic, the design has to offer unobtrusive access, optimum space utilization, and adaptabil- 
ity to user needs. The first studies in ergonomics were done in the USA at the start of industrialization, and it improved during the World Wars. Today, ergonomics is divided into two areas: reactive ergonomics (something will be fixed when it is necessary) and proactive ergonomics. Proactive ergonomics is the process of seeking areas that can be improved and fixed before problems occur.

It is in the area of proactive ergonomics that research has been performed to identify ergonomic aspects for elderly people. Elderly people adapt their environment in a way to serve their needs (e.g. items used less frequently are stored on the high shelf, whereas the things they need more frequently are stored at lower levels). Elderly people often forget they already have some items, which are then replaced and thus duplicated, resulting in a storage problem. Therefore, it is necessary to improve the accessibility to such items to reduce redundant storage space.

With this in mind, a robotic furniture system [5] has been designed. The system reduces space redundancy as the storage place is height adjustable. Through electrical motors and a rotational motion path (fig. 3 ), adjusting the height requires no physical strength from the user.

In addition to storing items in areas that are not easily reachable for them, eventually elderly people will also start to store things they do not frequently require in lower areas. With height-adjustable furniture, these limitations can be efficiently addressed. This concept can be applied in various areas within a home environment (e.g. apartment entrance, living room, kitchen, bathroom, etc.). When all required functions such as beds, sofas, tables, and chairs are integrated, it is estimated that elderly people can realize savings in terms of space of up to $20 \%$.

With this concept, a 1:1 partially functional prototype (fig. 4) has been realized. Electrical motors, controlled by a microprocessor, can transport each element via a chaintype belt, which is attached to the gear shaft of the motor. To allow a smooth vertical and horizontal translation and to secure the elements, a grid or rail-type mechanism is implemented. For the evaluation of this application, an age-simulation suit was used, and it simulated limitations of elderly people in performing simple movements. The prototype was evaluated in terms of ergonomy, organizational ergonomy within a room, and space use. The evaluation found that $20 \%$ less space is needed through the use of this concept, which would make room for wheelchairs or rollators.

A mini-PC is used to serve as the main processing unit. A series of sensors and devices are interfaced to the miniPC, such as a Microsoft Kinect sensor [15], an Arduino microcontroller board which controls the electric motors

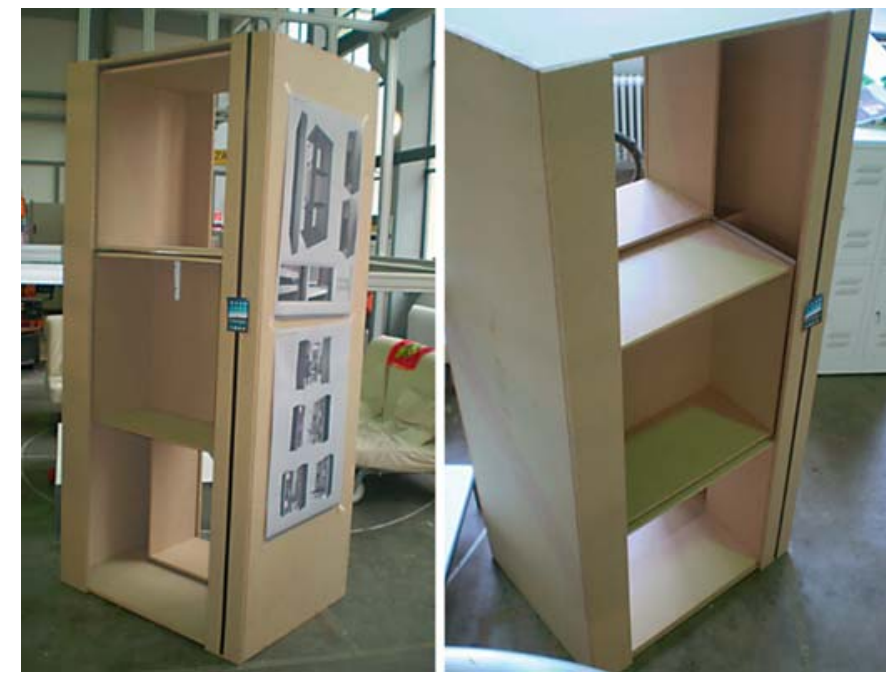

Fig. 4. 1:1 scale implemented prototype [5].

of the robotic furniture, a radio frequency identification (RFID) reader to check items in and out, a set of devices for monitoring vital signs [16], and a touch-screen tablet PC which provides the appropriate graphical user interface to allow the operation of the system features. The Kinect sensor was introduced to allow a certain degree of ambient intelligence since items stored in the robotic furniture are automatically logged upon entering the robotic furniture cabinets. The Kinect sensor offers a vertical tilt movement with a range of $\pm 27^{\circ}$ angles. By placing the sensor on top of the robotic furniture, the user can efficiently be detected upon approaching the furniture, thus signaling the system power-on, and also allowing it to detect items placed into the furniture cabinets. The tilt angle is automatically controlled by an algorithm which detects the user approach motion path, based on Kinect's skeletal tracking technique.

\section{Novel MiniOn Agent-Assisted Robotic Kitchen Platform}

Initially, information about basics of the modern kitchen, including the Frankfurt Kitchen and Workflow Analyses, was collected to develop a novel MiniOn agentassisted robotic kitchen platform [6] (fig. 5). The analyzed data can be divided into two main areas: (1) transformation in space and architecture (e.g. installing traditional Japanese sliding doors helps to alter the use and space of the room), and (2) optimization by multifunctionality 


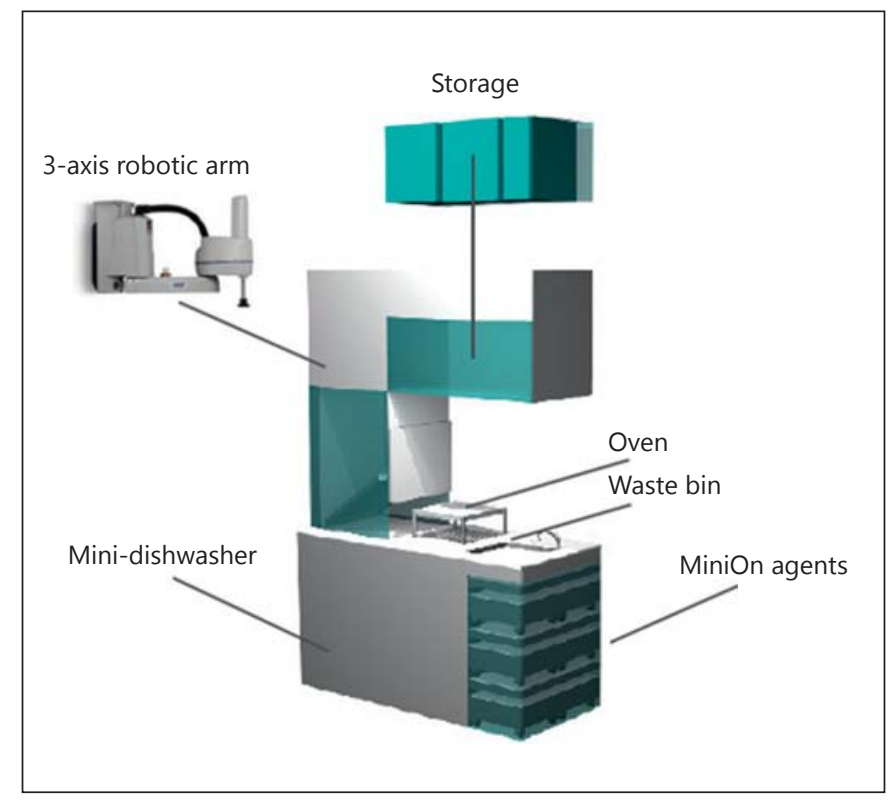

Fig. 5. Module design approach for the kitchen [6]. With permission, Sophia Kostova.

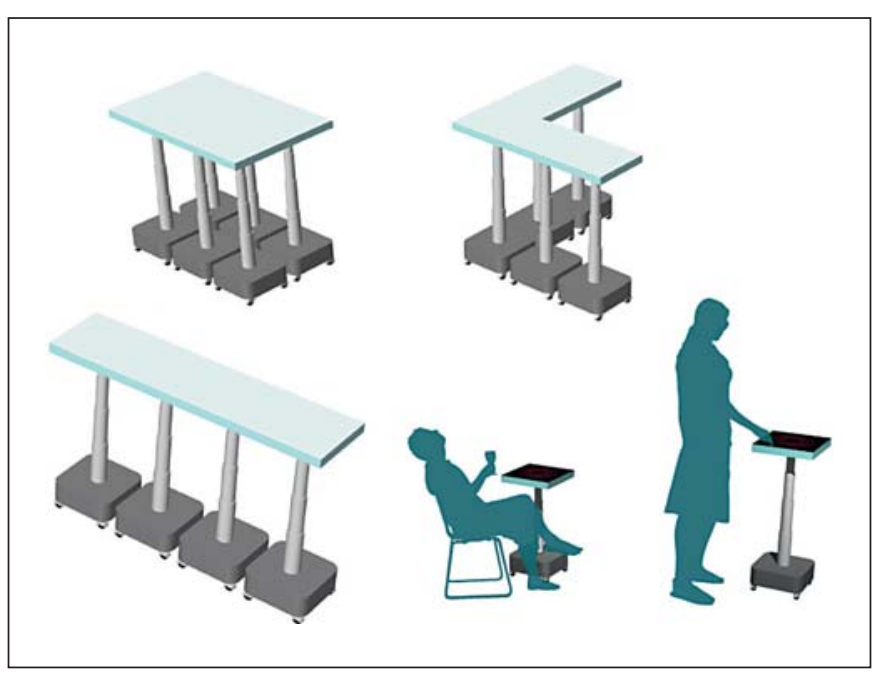

Fig. 6. Arrangement configurations of the MiniOn agents [6] With permission, Sophia Kostova.

(e.g. by using 'tatami' mats, special flooring material traditionally used in Japanese homes, the room can be used for several purposes such as a living room in the day time and a sleeping room at night). Therefore, an appropriate workspace needs to be planned. Furthermore, to increase the well-being of the user, personalization has to be considered.

This application also has the goal of supporting the user in his/her ADLs. As elderly people tend to spend most of their day in the kitchen, this proposed concept has an important influence for elderly people. As the lack of instant reflexes and fatigue increase with age, the danger of accidents also rises, especially in the kitchen. Through installing an intelligent and safe kitchen environment, this concept also reduces the chance of accidents.

The modern kitchen has been greatly influenced by the Frankfurt Kitchen, which was designed by Margarete Schütte-Lihotzky, with the aim to simplify the daily routine of housewives by rearranging the furniture. The workflow on which the Frankfurt Kitchen is based was developed by Fredrick Winslow Tailor, who analyzed and synthesized the workflows.

To investigate the workflow in the kitchens of elderly people, a study was prepared. The study included a real experiment in a real kitchen to identify the problems and limitations of elderly people. This experiment included cooking processes, cognitive support, and required space for each single step. For the cooking processes, the motion path of the person who is cooking was investigated. For the cognitive support experiment, a standard kitchen was modified to locate distraction factors and test cognitive support within the kitchen. To investigate the necessary space of a kitchen, the use of kitchen utensils was simulated (e.g. cutting vegetables), after the utensils were assessed on size, number, and frequency of use.

This experiment revealed that the person cooking faces problems while performing movements like bending down to the oven or standing still. Stiffness and pain force elderly people to a completely different approach compared to healthy people. This is why elderly people require more space in the kitchen. Therefore, the proposed concept minimizes the required space of the application. To support cognitive abilities, tags or pictures placed on the cabinet doors help to easily locate the appropriate utensil.

To create a flexible and efficient system, a modular concept was designed. The modules have three variations: fixed (necessary installation, e.g. sink, washing machine, etc.), semi-fixed (complicated to move, but moveable, e.g. integrated small electrical appliances, fridge, oven, etc.), and flexible (independent movement, e.g. storage, etc.). Furthermore, a 3 -axis robotic arm can assist in storing the utensils in their proper place, utilizing an electrostatic gripper. A compact dishwasher can help to save energy by analyzing the amount and degree of dirt on the dishes. A touchscreen is also installed, which is interfaced to a mini-PC. Over a Wi-Fi wireless interface, a vital sign measuring gateway is realized. This application 
Table 1. Limitations imposed in wheelchair functional transfers/ activities [7]

\begin{tabular}{|c|c|c|c|c|c|c|c|c|}
\hline \multirow[t]{2}{*}{ Area } & \multirow[t]{2}{*}{ Action } & \multicolumn{4}{|c|}{ Difficulty level } & \multicolumn{3}{|c|}{ Accident risk } \\
\hline & & $\mathrm{i}$ & s & & h & $\mathrm{s}$ & $\mathrm{n}$ & $\mathrm{h}$ \\
\hline \multirow{2}{*}{$\begin{array}{l}\text { Living } \\
\text { room }\end{array}$} & sofa/bed $\rightarrow$ wheelchair & $\mathrm{x}$ & & & & & & $\mathrm{x}$ \\
\hline & wheelchair $\rightarrow$ sofa/bed & $\mathrm{x}$ & & & & & & $\mathrm{x}$ \\
\hline \multirow[t]{9}{*}{ Kitchen } & shelf height, $\mathrm{cm}$ & & & & & & & \\
\hline & 10 & & & $\mathrm{x}$ & & & $\mathrm{x}$ & \\
\hline & 45 & & $\mathrm{x}$ & & & $\mathrm{x}$ & & \\
\hline & 80 & & $\mathrm{x}$ & & & $\mathrm{x}$ & & \\
\hline & 125 & & $\mathrm{x}$ & & & $\mathrm{x}$ & & \\
\hline & $>160$ & $\mathrm{x}$ & & & & $\mathrm{x}$ & & \\
\hline & sink & & & & $\mathrm{x}$ & $\mathrm{x}$ & & \\
\hline & water tap & $\mathrm{x}$ & & & & $\mathrm{x}$ & & \\
\hline & kitchen worktop & & & & $\mathrm{x}$ & $\mathrm{x}$ & & \\
\hline \multirow{4}{*}{$\begin{array}{l}\text { Dining/ } \\
\text { working } \\
\text { room }\end{array}$} & $\begin{array}{l}\text { move the wheelchair } \\
\text { towards table }\end{array}$ & & $\mathrm{x}$ & & & $\mathrm{X}$ & & \\
\hline & move the wheelchair & & & & & & & \\
\hline & away from table & & $\mathrm{x}$ & & & $\mathrm{x}$ & & \\
\hline & eat and work & & $\mathrm{x}$ & & & $\mathrm{x}$ & & \\
\hline
\end{tabular}

can also make use of the telemonitoring devices [16] (blood pressure meter, blood glucose level meter, and scale that measures BMI) of the Vision Controlled Robotic Furniture System [5]. Therefore, the user can wirelessly monitor his/her own health status. The health status can be sent via telecare and telemedicine to a physician. Thus, elderly people who are unable to leave their own home can receive professional healthcare.

The user can adapt the MiniOn (fig. 6) robotic agent, which consists of a square plate that is attached to a telescopic base into a mobile platform, to their needs, e.g. to assist in cooking, serving, and cleaning up.

Autonomous navigation of the MiniOn is achieved by optical sensors with depth-estimation capabilities [15]. To enable the MiniOn with autonomous navigation, the exact space arrangement must be known. To do this, the simultaneous localization and mapping [17] technique is used. This technique is used by autonomous robots to build up/update a map in an unknown area, while at the same time keeping track of their current location. Each MiniOn can autonomously navigate in order to dock itself to different types of walls or furniture. This is performed using label stickers, attached to the designated locations. The mobile robots scan the surrounding area using images acquired by their onboard optical sensor, and once the appropriate sticker pattern is detected with-

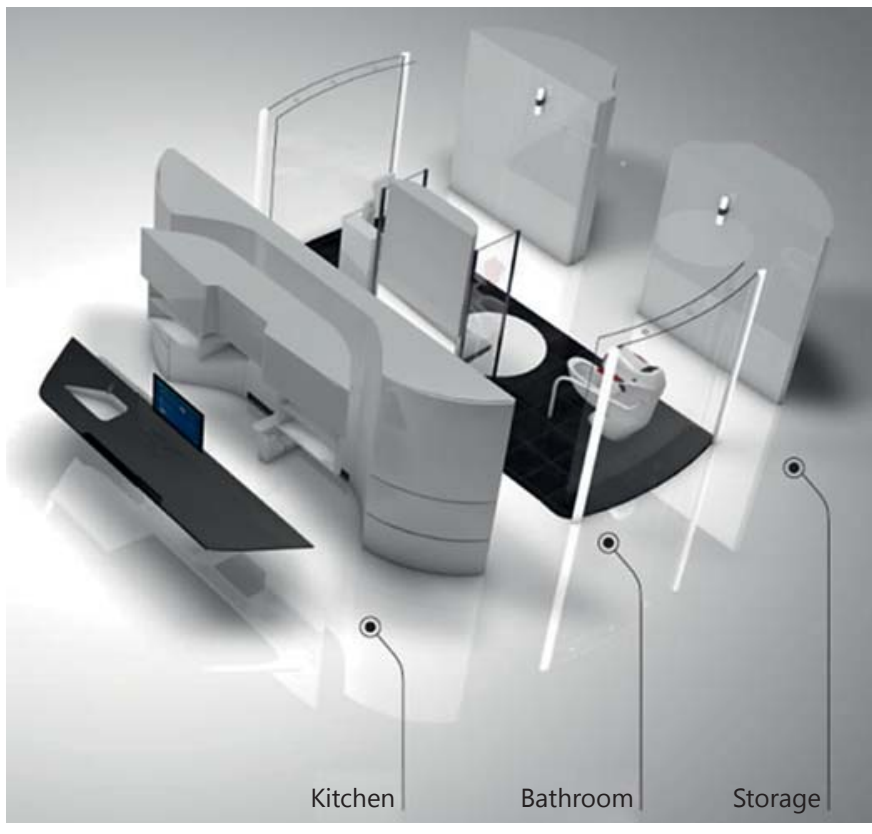

Fig. 7. Functions of the proposed concept [7]. With permission, Hai Ninh.

in the optical scene, they are visually guided towards their target. A similar approach is used in order to be linked against each other to form the desired configurations.

\section{Coadaptation of Assistive Mobility Devices and Residential Functions}

Basic ADLs (BADLs) [18] consist of self-care tasks, including personal hygiene and grooming, dressing and undressing, self-feeding, functional transfers (from wheelchair to bed/toilet, etc.), and ambulation (walking without the use of assistive devices). Instrumental ADLs are as important as BADLs, but they allow individuals to live independently in a community [19], e.g. doing housework, taking prescribed medications, managing money, shopping for groceries or clothing, using a telephone or other forms of communication, using technology, and using transportation within the community. Today there are a lot of subsequent developments for supporting and assisting the ageing society in BADLs and instrumental ADLs. However, acceptance is often negligible by the majority of elderly people due to the high costs and the complexity of the technology. A solution could be to optimize the wheelchair design and to modify the environment arrangement. 
Fig. 8. Social concept: floor plan is used for viewpoint of the concept [7]. With permission, Hai Ninh.

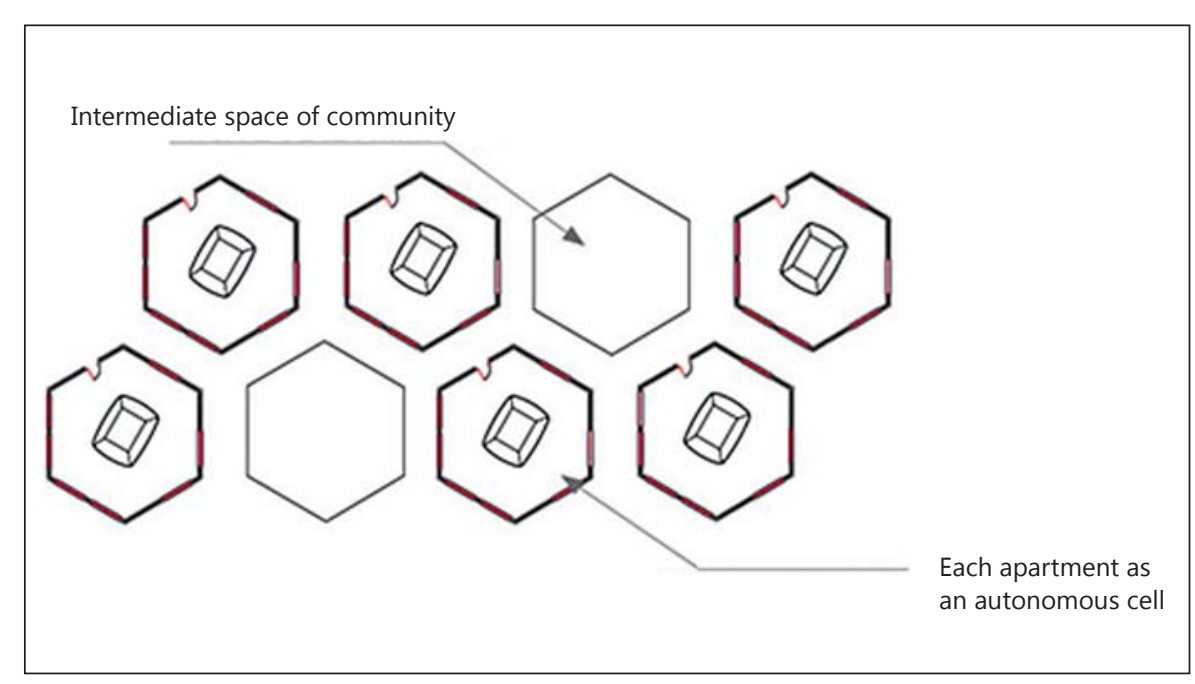

To identify the difficulties and limitations which occur with ADLs, experiments in the living room, kitchen, and dining/working room have been performed. For the living room, the transfer from a wheelchair to a sofa was tested. Both the difference in height and the armrest pose difficult challenges for elderly people in moving from the wheelchair to the sofa. In the kitchen, the standardized dimensions show a lot of disadvantages for a person in a wheelchair when no adaptions have been made, e.g. if there is no free space under the kitchen worktop. In the dining/working room, a limitation was found in the height of the table as it is not possible to sit at the table (standard height) in a wheelchair. Therefore, the range for the effective use of the table decreases, e.g. by eating or working. Table 1 lists these problems, which are mostly due to dimensions.

Based on these results, a mock-up of an optimized wheelchair was designed and a concept was developed (fig. 7) consisting of three parts: architecture, social, and networking. In the architectural concept, it is planned to summarize and structure the functions of a room.

By placing functions together, e.g. kitchen and bath, the separating wall in between becomes redundant and a new type of cell structure is developed. In that way most of an apartment space can be fused, while the rest stays barrier-free.

In the networking concept, it is planned to install services like telecare, domestic aid, security, infotainment, databases, and information exchange platforms. The social concept aims to promote the social connection of elderly people. In case of disabilities or health conditions, the only social contact is the telephone. The current gen-

Towards a Future Robotic Home

Environment: A Survey eration may be weary of the World Wide Web and not utilize it, although it is believed that this will not be an issue for subsequent generations of elderly people. While social networks will become a solution in the future, currently the ground plan for designing apartments for elderly people should consider two main types: private and public areas. A harmonic suggestion is illustrated in figure 8.

To install this concept into an apartment, unnecessary partition walls have to be removed. The ADLs will completely focus on the main cell. A specially designed wheelchair ensures the best possible mobility within the cell. Integrated sensors communicate with the cell and exchange acquired data.

Optimized furniture supports the independence of the user. As the cell will be modular, it can be individualized according to the individual needs, and in this way the transition ways can be shortened. A central rotating platform mechanism embedded in the floor supports the mobility of a person in a wheelchair by rotating the user to the desired function of the bathroom. The wheelchair itself consists of three main components: substructure, central axis, and seat. The sensors (mainly for vital sign measuring) are integrated within the armrest, e.g. pulse oximeter and electrocardiogram sensor. Furthermore, it is planned to incorporate a respiration frequency meter, weight analysis meter (for measuring BMI), and an activity logger sensor (on and around the chair). Based on this research, a multidisciplinary consortium has been created involving academic and industry partners. The consortium is researching in the field of AAL, and the project is called PASSAge. 
Table 2. Bathing time requirement evaluation results

\begin{tabular}{lllc}
\hline Person & Age & Sex & $\begin{array}{c}\text { Bathing time } \\
(\text { min })\end{array}$ \\
\hline 1 & 23 & female & 6.3 \\
2 & 25 & male & 4.7 \\
3 & 26 & female & 9.5 \\
4 & 51 & female & 12.3 \\
5 & 56 & male & 5.4 \\
6 & 79 & female & 15.2 \\
7 & 83 & female & 13.3 \\
8 & 85 & male & 14.2 \\
\hline
\end{tabular}

\section{Concept for Assisting and Automating Daily Activities in the Bathroom}

BADLs consist of self-care tasks, self-feeding, functional transfers, and mobility. Therefore, bathing, dressing, and eating can be used to assess the independence of elderly people. For this category, numerous assistive devices have been developed. The design of these devices focuses on simplifying hygiene tasks because the sense of shame is very strong in such situations. The disadvantage of such applications is that there is still a caretaker needed to assist or to supervise the utilization. For example 'walkin tubs' are bathtubs with an integrated door, with the advantage that there is no heightened side edge, which is an obstacle for elderly people.

Additional tasks were evaluated during the experiment. According to gender, the following tasks were performed: removing clothes, using the toilet, brushing teeth, showering, hair washing, shaving, hand washing, drying off, hair styling, blow drying, cleaning ears, dressing, face washing, putting on lotion, putting on cosmetics, and washing with a washcloth [8]. The results can be summarized in three main identified points: (1) usage versus layout - scattered processes and long distances between every 'station', (2) agility - physical agility decreases in old age and complicates the daily routine, and (3) mobility - confined spaces affect domestic mobility. The experiment also confirmed that elderly persons require much longer for the entire bathing process, i.e. undressing, showering, drying off, and getting dressed (table 2).

After evaluating the results, a concept for optimizing the bathroom was developed, with the focus set on mobility and efficiency. As the average single-family house in Europe [20] is around $60 \mathrm{~m}^{2}$, space must be utilized as

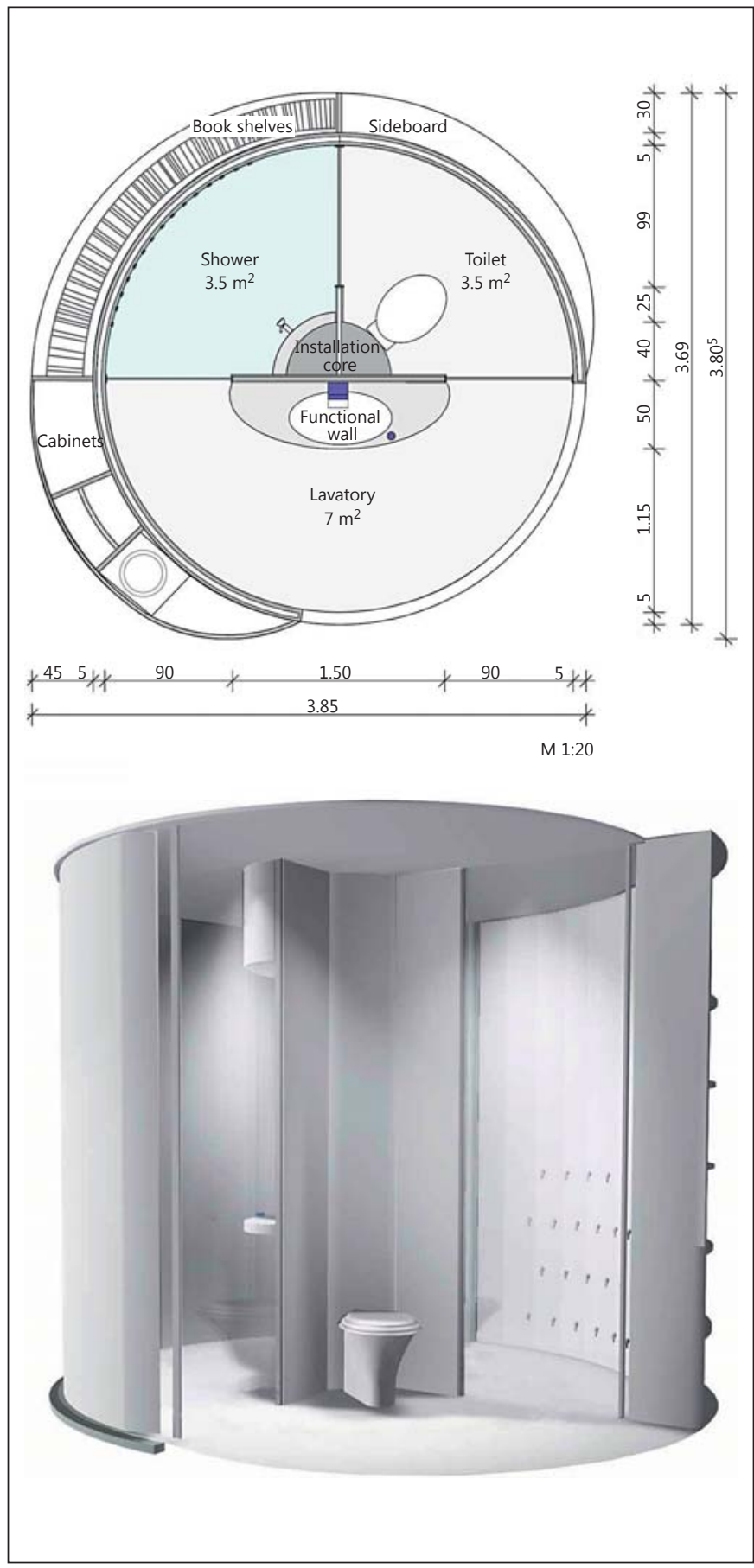

Fig. 9. Proposed bathroom environment (floor plan) [8]. With permission, Mira Semmer.

efficiently as possible. To improve the process flow of BADLs, compared to the bath environment of a normal apartment, a single cell will fuse all necessary steps. The cell will be able to offer this kind of service by introducing various technologies and robotic elements. For ex- 
ample, an arrangement of a drivable toilet will allow that a wheelchair user can transfer straightforward without needing extreme strength. By embedding cabinets, important space can be saved. Services like telecare and teleconsulting can be embedded in the bathroom in a functional wall behind the mirror. A mobile assistive wheelchair, which is able to adapt to the various functions of the bathroom, simplifies use by offering conformity and enhancing the independence of elderly people. To receive a barrier-free arrangement, the layout has to be flexible.

Therefore, the proposed futuristic bathroom cell will be divided into three sections, as shown in figure 9: shower room, toilet, and functional wall with a washbasin. The shower room has integrated nozzles in the wall which spray water and shower gel. After the shower is finished, integrated blowers dry the user with hot air. The storage cabinet, shelves, and washbasin are heightadjustable. An electric wheelchair ensures the user's mobility. The seat of the chair can rise to each necessary level, and thus the wheelchair can be used for most of the activities inside the cell. As a result, repeat transfers are not required [8].

\section{Deploying Mechatronics and Robotics in Architecture}

Environments and prototypes have been developed and designed for smart building since the 1980s, e.g. the Tron House 1, 2, and 3 [21], US Aware Home [22], PlaceLab [23], and MIT's House_n [24]. Prototypes of assistive homes have also been built in Germany [e.g. 'Haus der Gegenwart' (house of the present) [25] and 'Haus der Zukunft' (house of the future) [26]]. These prototypes include sensor-actuator systems, but mostly these are not designed to support the ADLs of elderly people. Considering the idea of AAL, the main focus will be placed on health monitoring and patient-physician communication in the future. Therefore, the integration of digital communication frameworks has been investigated in many projects, whereas the integration of robotic systems has been neglected. Thus, the proposed concept considers architectonic integration and combines elements of smart building, robotic rooms, and AAL technologies.

Since 2008, subsystems have been investigated and tested by an interdisciplinary team of researchers in order to develop seamless robotic assistance. Some of the subsystems are cited in table 3. Figure 10 shows eight

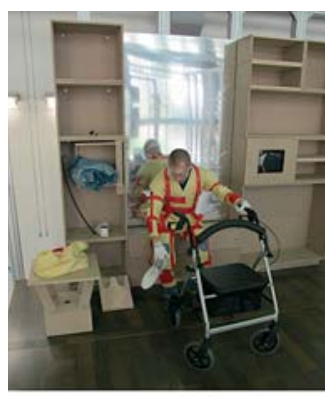

Robotic service wall

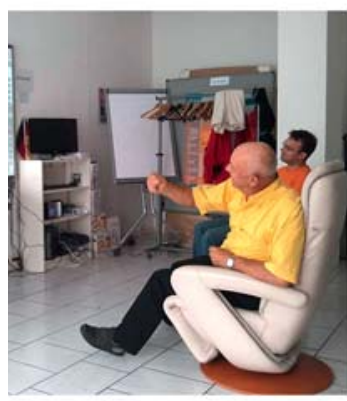

Robotic chair

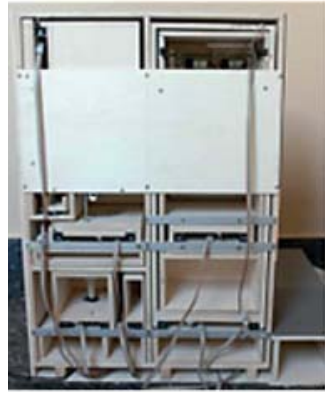

Robotic service core

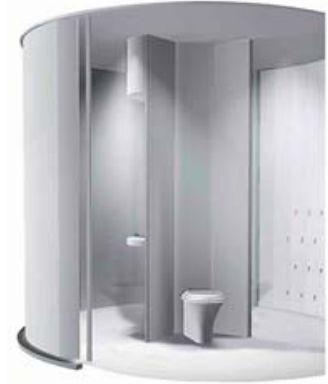

Automated bathroom

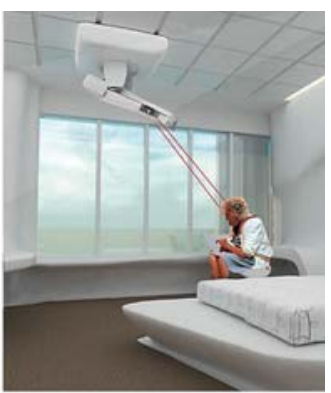

Modular ceiling robot concept

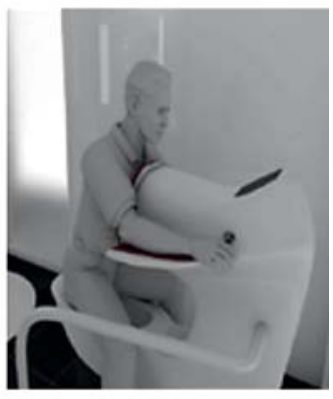

Robotic in-house transfer

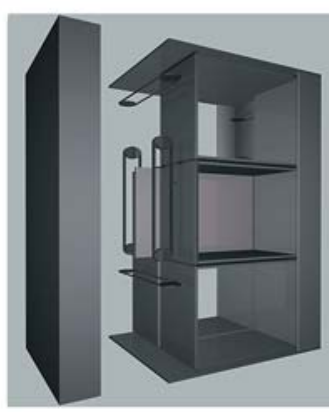

Robotic cabinets

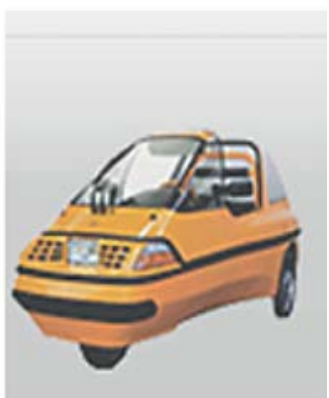

Combined indooroutdoor mobility
Fig. 10. Overview of experimental approaches according to categories [9]. 


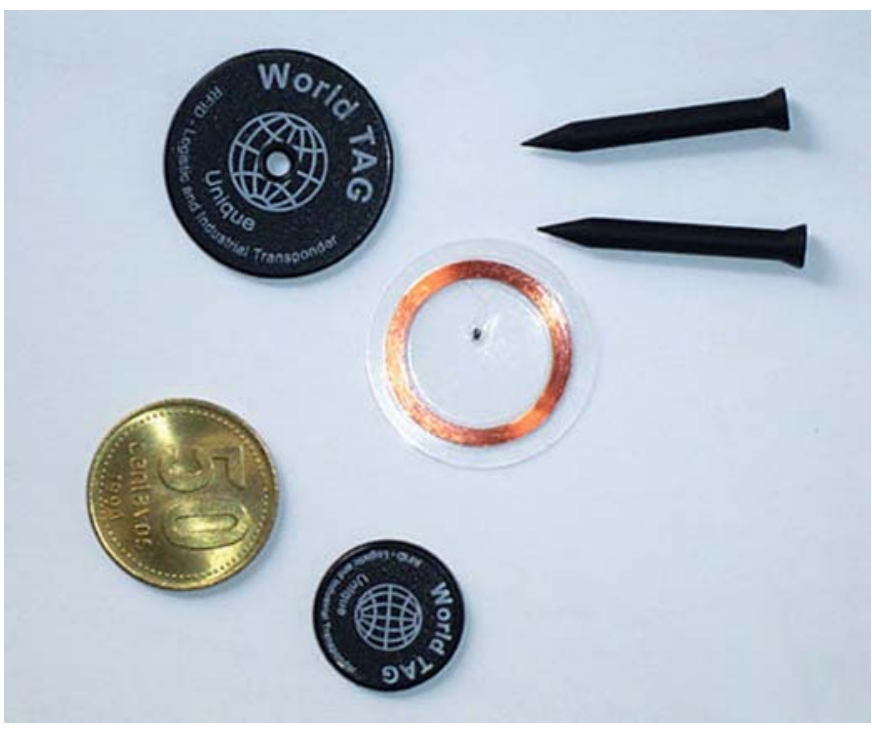

Fig. 11. RFID tags [9].

different devices developed to assist elderly people in their home environment. Through these applications, the independence of elderly people should increase, as they are not dependent on a caretaker in order to perform BADLs.

The robotic service wall has been implemented in 'Living Independently in South Tyrol Alto Adige' (LISA; July 2010 to July 2012), a 2-year funded research project. The consortium consists mainly of industry partners, with the Technical University of Munich as the center point of the consortium both in terms of strategy and technology development. In LISA, the implementation of a novel robotic service wall to support ADLs is the main objective. The proposed system followed a modular approach, whereas all system elements provide 'plug and play' characteristics. Robotic actuators, sensors, and display screens are populating the proposed robotic wall, in order to enable an AAL approach.

The implementation of the robotic chair is supported by a funded research and development project in the field of AAL called GEWOS. The main objective was to develop a chair that is equipped with invisible but sophisticated sensor technology that measures a multitude of the user's vital signs. These inconspicuously and at first glance invisibly integrated sensor modules form the basis for professional activity and sports concepts, health assistance, and business and service concepts that are delivered as 'services' through the chair and its interface as a channel to the users, which through the sensor systems
Table 3. Overview of proposed experimental approaches according to categories [8]

\begin{tabular}{|c|c|c|c|}
\hline \multirow[t]{2}{*}{ Subsystem } & \multicolumn{3}{|l|}{ Phase } \\
\hline & $\begin{array}{l}\text { step 1: } \\
2008-2011\end{array}$ & $\begin{array}{l}\text { step 2: } \\
2011-2012\end{array}$ & $\begin{array}{l}\text { step 3: } \\
2012\end{array}$ \\
\hline Wall & $\begin{array}{l}\text { robotic service wall - } \\
\text { research project LISA }\end{array}$ & & \\
\hline Ceiling & modular ceiling robot & & \\
\hline Service core & robotic service core & & \\
\hline Cabinet & robotic cabinets & & \\
\hline Seat & $\begin{array}{l}\text { robotic chair - research } \\
\text { project GEWOS }\end{array}$ & & \\
\hline In-house mobility & robotic in-house transfer & & \\
\hline Bathroom & & $\begin{array}{l}\text { automated } \\
\text { bathroom }\end{array}$ & \\
\hline $\begin{array}{l}\text { Combined indoor/ } \\
\text { outdoor mobility }\end{array}$ & & $\begin{array}{l}\text { research project: } \\
\text { PASSAge }\end{array}$ & \\
\hline $\begin{array}{l}\text { Integrated robotic } \\
\text { environment }\end{array}$ & & & AIR LAF \\
\hline
\end{tabular}

actually becomes part of the system. Therefore, GEWOS considers the sensor chair as a sociotechnical system, comprising an internet-based platform, an interface component, and further interaction elements and matching services. By encouraging movement and supporting further constitutional methods, this system serves as a health promoter, embedded in the physical and emotional surroundings of the user.

The proposed robotic in-house transfer approach identified the needs and requirements elderly people face in performing ADLs by conducting a survey among various age groups and identifying the main bottlenecks and limitations. A design procedure was then proposed in order to provide an efficient solution for optimizing independence in functional transfers (getting in and out of a bed or wheelchair, getting on or off the toilet, etc.) of elderly people within the home environment. The proposed concept comprises the combination of robotic and architectural elements, aiming at the realization of a novel indoor mobility device enabling independent living, efficiently addressing the mobility difficulties elderly people face within the home environment.

The prefabricated robotic service core is a unit which could be integrated into apartments as a 'room within a room' system. With its standardized height of $235 \mathrm{~cm}$, it could be positioned in an apartment in an old building as well as in a newly built house. This system offers services to handicapped people. Only minimum movements are required to perform ADLs, as the bath and 
kitchen are ergonomically reachable from the bed. Within the unit the vital data can be continually measured. Via an integrated communication screen, physicians and the individual can communicate. Therefore, this unit represents a small hospital within the home environment. The cell is realized by the fusion of functional architecture, embedded microcontrollers, and ambient intelligence technologies in order to provide disabled individuals the possibility to stay at home as long as possible.

The implementation of combined indoor-outdoor mobility (funded research and development project) is taking shape with the PASSAge project. The goal of the project is to ensure the mobility of elderly people by developing a mobility system that can be integrated into the surroundings of the individual. Furthermore, the solution will enhance the quality of living by supporting the safety, comfort, and health of the individual. This will be realized by user-friendly add-on modules.

The existing Building Information Modeling [27] needs to integrate sensors that in the future will enable the user to interact with the home environment. Since a wide range of sensors is necessary, a middleware, like the Robot Operating System (ROS) [28], is necessary in order to access and control the environment. ROS is able to handle the data information exchange in real-time. Combined with a $3 \mathrm{D}$ visualization simulator environment called $\mathrm{Ga}$ zebo [29], it is possible to model a ubiquitous environment by considering that the building is an immobile robot with a lot of links.

Today's RFID technology enables identification from a distance without requiring any line pattern tags (like bar codes) for detection [30]. At the same time, RFID is able to store more than ID information, e.g. manufacturer, product type, etc. RFID can be divided into active and passive RFID sensors [31]. The difference between these two sensor categories is that an active RFID (e.g. transponder) needs a power supply. The passive RFID consists of three parts: antenna, processing unit, and encapsulation. The power supply for the processing unit is captured by the antenna, which also reads and transfers the tags ID to the tag reader.

In a home environment, this technology can be utilized to assist elderly people by providing them a realtime inventory of their high-priority items. Experiments conducted during the proposed study revealed the efficiency of this 'invisible' technology (because of the size of the tags, see fig. 11) and the variety of potential applications that this technology can contribute to. By combining computerized databases and inventory control, linked through digital communication networks spread across the home environment and across a global set of locations, RFID technology can efficiently pinpoint individual items as they move between locations, warehouses, vehicles, and stores.

\section{Conclusion and Future Work}

Several concepts to support elderly people in their ADLs and BADLs have been proposed. The concepts differ in various areas such as ceiling suspended systems (MRAS), automated shelves in order to achieve storage place and ergonomy for elderly people, new kitchen designs with MiniOn robotic mobile agents which offer a dynamic environment realization, a robotic home environment, and an automated bathroom in combination with a novel robotic wheelchair concept. By introducing new technologies into the home environment like robots (e.g. Lynx, TurtleBot, etc.) and sensors (Microsoft Kinect, RFID, etc.), smart homes and flats can be realized.

The aim of this research is to create smart environments to assist elderly and disabled people in their ADLs through preservation of their independence. The integration and permeation of our environments and social spaces with robotic technology and related subsystems will lead to new concepts and explore new forms such as robotic walls, robotic furniture, robotic care environments, mobility robots, and robotic everyday companions. Obviously, emerging technologies are not only transforming the functionality of upcoming hybrid robot environment systems, but also designs and forms of our environment.

Despite all of the complex interdependencies and transformations, underlying principles like modularity, customization capability, system distribution, standardization, and a well-defined human-robot relation seem to be helpful tools in designing future hybrid robot-environment systems. Furthermore, modularity and standardization are important prerequisites for industrially fabricated and customized robotic enhanced assistive environments. Complex and multilevel high-tech environments will only achieve affordability and long-term serviceability when fabricated with the latest industrial technology. 


\section{References}

$\checkmark 1$ Cohen JE: Human population: the next half century. Science 2003;302:1172-1175.

-2 Wiener JM, Hanley RJ, Clark R, Van Nostra JF: Measuring the activities of daily living: comparisons across national surveys. J Gerontol 1990;46:229-237.

3 ArjoHuntleigh Ceiling Lift. http://www. arjohuntleigh.com/ukah/Products.asp?Page Number $=3604 \&$ ProductCategory_Id=15.

4 Linner T, Georgoulas C, Bock T: A multi-robotic assistant system (MRAS): a development approach with application to the ageing society. Gerontechnology 2012;11:381.

$\checkmark 5$ Georgoulas C, Linner T, Bock T: Vision controlled robotic furniture system. Gerontechnology 2012;11:370.

6 Georgoulas C, Linner T, Bock T: A novel MiniOn agent assisted robotic kitchen platform. Gerontechnology 2012;11:369.

$>7$ Bock T, Linner T, Georgoulas C: Co-adaptation of assistive mobility devices and residential functions. Gerontechnology 2012;11:363.

$>8$ Bock T, Georgoulas C, Linner T: Towards robotic assisted hygienic services: Concept for assisting and automating daily activities in the bathroom. Gerontechnology 2012;11:362.

$>9$ Linner T, Georgoulas C, Bock T: Advanced building engineering: deploying mechatronics and robotics in architecture. Gerontechnology 2012;11:380.

$>10$ Murray D, Little JJ: Using real-time stereo vision for mobile robot navigation. J Auton Robots $2000 ; 8: 161-171$.

11 Jung JW, Do JH, Kim YM, Suh KS, Kim DJ, Bien ZZ: Advanced robotic residence for the elderly/the handicapped: realization and user evaluation; in: Rehabilitation Robotics. 9th International Conference on Rehabilitation Robotics. Piscataway, IEEE, 2005, pp 492 495.
12 Sato T, Harada T, Mori T: Environment-type robot system. Robotic room featured by behavior media, behavior contents, and behavior adaptation. IEEE Trans Mechatronics 2004;9:529-534.

13 Waseda University, Wabot House. http:// www.wabot-house.waseda.ac.jp/html/e-top. htm.

14 Murakami K, Hasegawa T, Karazume R, Kimu-ro Y: A structured environment with sensor networks for intelligent robots. IEEE Sensors 2008;705-708.

15 Microsoft Kinect. http://en.wikipedia.org/ wiki/Kinect.

16 Biocomfort Health Manager Platform. http:// www.biocomfort.de/telemonitoring_devices_overview.html.

17 Dissanayake MW, Newman P, Clark S, Durrant-Whyte HF, Csorba M: A solution to the simultaneous localization and map building (SLAM) problem. Robotics and automation. IEEE Trans 2001;17:229-241.

18 McDowell I, Newell C, Measuring Health: A Guide to Rating Scales and Questionnaires, ed 2. New York, Oxford University Press, 1996.

19 Bookman A, Harrington M, Pass L, Reisner E: Family Caregiver Handbook. Cambridge, MIT, 2007.

20 United Nations Publication: Trends in Europe and North America: The Statistical Yearbook of the Economic Commission for Europe (Trends in Europe and North America). New York, UNO, 2001.

21 Shimizu N: A house of sustainability: PAPI intelligent house in the age of ubiquitous computing. Architecture and Urbanism, Dec 2005 (special issue).
22 Kidd CD, Orr R, Abowd GD, Atkeson CG, Essa IA, MacIntyre B, Mynatt ED, Starner T, Newstetter W: The aware home: a living laboratory for ubiquitous computing research; in: Proceedings of the Second International Workshop on Cooperative Buildings, Integrating Information, Organization, and Architecture. London, Springer, 1999, pp 191198.

23 Intille S, Larson K, Tapia EM, Beaudin J, Kaushik P, Nawyn J, Rockinson R: Using a live-in laboratory for ubiquitous computing research; in: PERVASICE '06 Proceedings of the 4th International Conference on Pervasive Computing. Heidelberg, Springer, 2006, pp 349-365.

24 Larson K, Stephen I: MIT Open Source Building Alliance - A House_n Initiative. Position Paper. Cambridge, MIT House_n, 2005.

25 Haus der Gegenwart, Munich, Germany. Partners: Microsoft, BMW, Munich City. http://www.haus-der-gegenwart.de/partner/ (accessed November 12, 2009).

26 Intelligent Networking: T-Com House. Berlin, Siemens, Telecom Laboratories, 20052006.

27 Eastman C, Teicholz P, Sacks R, Liston L: BIM Handbook - A Guide to Building Information Modeling for Owners, Managers, Architects, Engineers, Contractors and Fabricators. Hoboken, John Wiley \& Sons, 2008.

28 ROS. http://www.ros.org/wiki/ROS.

29 Gazebo. http://gazebosim.org/about.html.

30 Finkelzeller K: The RFID Handbook, ed 2. Hoboken, John Wiley \& Sons, 2003.

31 Want R: An introduction to RFID technology. IEEE Pervasive Comput 2006;5:25-33. 